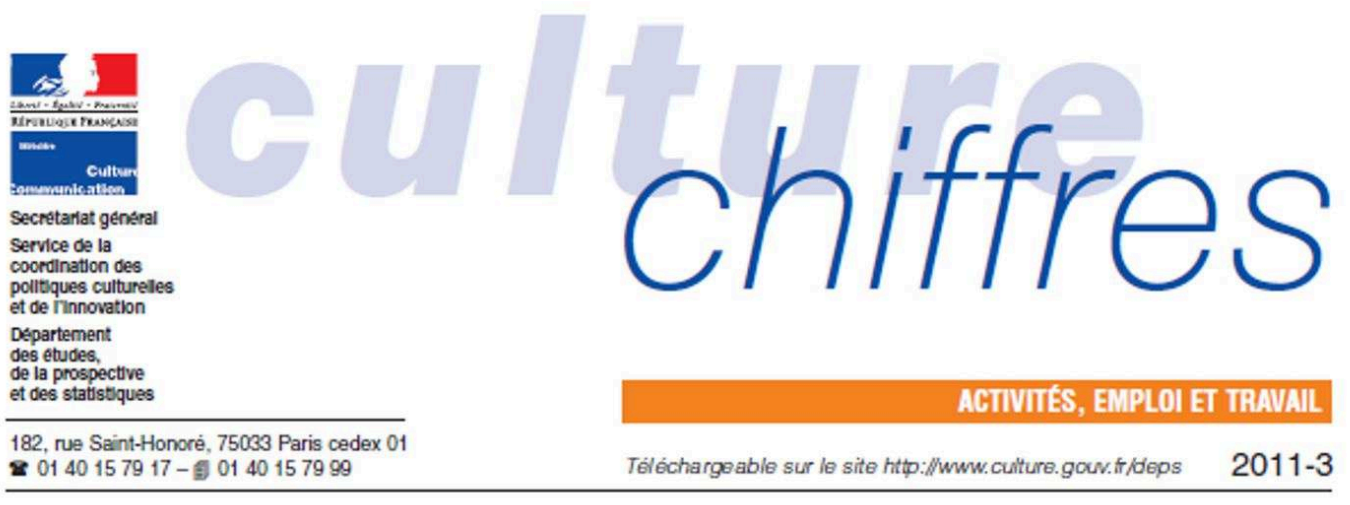

\title{
Écrivains, photographes, compositeurs... Les artistes auteurs affiliés à l'Agessa en 2008"
}

Marie GouYon**
Writers, photographers, composers... Artist-author members of AGESSA in 2008
De plus en plus d'artistes auteurs affiliés

En 2008, l'Agessa rassemble 11000 artistes auteurs affiliés (encadré, p. 2), répartis sur onze disciplines. Les plus nombreux sont les auteurs du livre - avec 4300 écrivains, illustrateurs et traducteurs - suivis des photographes (3450) et des auteurs d'œuvres audiovisuelles ${ }^{1}$ (un peu plus de 1800) (tableau 1).

Les effectifs d'artistes auteurs ont augmenté de $58 \%$ en 15 ans (ils étaient 7000 en 1994). Cette progression est principalement due aux auteurs d'œuvres audiovisuelles (y compris cinématographiques) dont le nombre a doublé au cours de la période, aux photographes $(+83 \%)$, aux illustrateurs $(+79 \%)$ et aux traducteurs $(+64 \%)$. Certaines populations d'artistes auteurs se sont au contraire contractées : celles des auteurs d'œuvres dramatiques $(-16 \%)$ et des auteurs de compositions musicales $(-13 \%)$ notamment (graphique 1).

Une population qui se féminise, inégalement selon les disciplines

En 2008, deux artistes auteurs sur trois sont des hommes. Cette surreprésentation masculine est nettement plus marquée que dans l'ensemble de la population active en emploi, composée à $53 \%$ d'hommes ${ }^{2}$.
Tableau 1 - Répartition des artistes auteurs affiliés à l'Agessa selon la discipline en $2008^{3}$

\begin{tabular}{|c|c|c|}
\hline Disciplines** & Nombre & $\%$ \\
\hline Photographes & 3447 & 31 \\
\hline Ecrivains & 2038 & 18 \\
\hline Auteurs d'œuvres audiovisuelles & 1835 & 17 \\
\hline Illustrateurs & 1388 & 13 \\
\hline \multicolumn{3}{|l|}{ Auteurs de compositions musicales } \\
\hline avec ou sans paroles & 877 & 8 \\
\hline Traducteurs & 876 & 8 \\
\hline Auteurs du multimedia interactif*... & 270 & 2 \\
\hline Auteurs de logiciels & 146 & 1 \\
\hline Auteurs d'œuvres dramatiques & 132 & 1 \\
\hline Choregraphes & 9 & - \\
\hline Ensemble & 11018 & 100 \\
\hline \multicolumn{3}{|c|}{  } \\
\hline
\end{tabular}

Si la traduction est une discipline aux deux tiers féminine, toutes les autres sont majoritairement masculines, dans des mesures variables: en 2008 , les femmes représentent entre $40 \%$ et $50 \%$ des écrivains et des auteurs

* Cette étude a bénéficié du concours actif de Pascal Murcans, Thierry Dumus et Samba Duciñ, respectivement chef du département des artistes et des professions à la Direction générale de la création artistique du ministère de la Culture et de la Communication, directeur de l'Agessa et chef de projet informatique à I'Agessa. Qu'ils soient ici vivement remerciés.

projet informatique à I A gessa. Qu 'ils soient ici vivement remercics

1. En 2006, la catégorie des auteurs d'œuvres cinématographiques (200en 2005) a été agrégée à celle des auteurs d'œuvres audiovisuelles. Les auteurs 1. En 2006 , la catégorie des auteurs d'cuvres cinématographiques (200en 2005 ) a été agrégée à celle des auteurs d' 'auvres audiovisuel
d'auvres audiovisuelles (y compris cinématographiques) sont des réalisateurs, des scénaristes, des dialoguistes, des sous-titreurs, etc. 2. Selon l'enquête Emploi 2008 de l'Insee.

3. La présente étude porte sur les seuls artistes auteurs affiliés à l'Agessa, avec un bref exposé, en encadré, de la situation des assujettis (précomptés). 


\section{Écrivains, photographes, compositeurs...}

Les artistes auteurs affiliés à l'Agessa en 2008

Writers, photographers, composers... Artist-author members of AGESSA in 2008

\section{Marie Gouyon}

Éditeur : Département des études, de la prospective et des statistiques

Lieu d'édition : Paris

Année d'édition : 2011

Date de mise en ligne : 21 septembre 2015

Collection : Culture chiffres

ISBN électronique : 9782111398559

\section{Qbooks}

http://books.openedition.org

\section{Édition imprimée}

Date de publication : 1 avril 2011

Nombre de pages : 8

\section{Référence électronique}

GOUYON, Marie. Écrivains, photographes, compositeurs... : Les artistes auteurs affiliés à l'Agessa en 2008. Nouvelle édition [en ligne]. Paris : Département des études, de la prospective et des statistiques, 2011 (généré le 25 avril 2021). Disponible sur Internet : <http://books.openedition.org/deps/532>. ISBN : 9782111398559. 

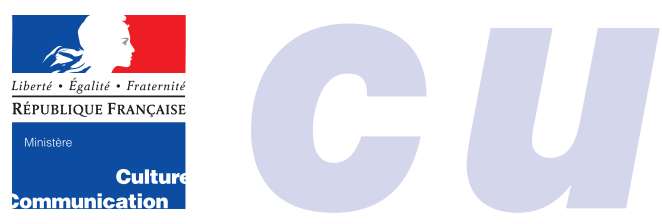

Secrétariat général

Service de la

coordination des

politiques culturelles

et de l'innovation

Département

des études,

de la prospective

et des statistiques

\section{Écrivains, photographes, compositeurs... Les artistes auteurs affiliés à l'Agessa en 2008 "}

\author{
Marie GouYon**
}

\section{Writers, photographers, composers... Artist-author members of AGESSA in 2008}

\section{De plus en plus d'artistes auteurs affiliés}

En 2008, l'Agessa rassemble 11000 artistes auteurs affiliés (encadré, p. 2), répartis sur onze disciplines. Les plus nombreux sont les auteurs du livre - avec 4300 écrivains, illustrateurs et traducteurs - suivis des photographes (3 450) et des auteurs d'œuvres audiovisuelles ${ }^{1}$ (un peu plus de 1800) (tableau 1).

Les effectifs d'artistes auteurs ont augmenté de $58 \%$ en 15 ans (ils étaient 7000 en 1994). Cette progression est principalement due aux auteurs d'œuvres audiovisuelles (y compris cinématographiques) dont le nombre a doublé au cours de la période, aux photographes $(+83 \%)$, aux illustrateurs $(+79 \%)$ et aux traducteurs $(+64 \%)$. Certaines populations d'artistes auteurs se sont au contraire contractées : celles des auteurs d'œuvres dramatiques $(-16 \%)$ et des auteurs de compositions musicales $(-13 \%)$ notamment (graphique 1).

\section{Une population qui se féminise, inégalement selon les disciplines}

En 2008, deux artistes auteurs sur trois sont des hommes. Cette surreprésentation masculine est nettement plus marquée que dans l'ensemble de la population active en emploi, composée à $53 \%$ d'hommes $^{2}$.

\section{Tableau 1 - Répartition des artistes auteurs affiliés à l'Agessa selon la discipline en $2008^{3}$}

\begin{tabular}{lcr|}
\hline Disciplines $^{* *}$ & Nombre & $\%$ \\
Photographes & 3447 & 31 \\
Écrivains & 2038 & 18 \\
Auteurs d'œuvres audiovisuelles & 1835 & 17 \\
Illustrateurs & 1388 & 13 \\
Auteurs de compositions musicales & & \\
$\quad$ avec ou sans paroles & 877 & 8 \\
Traducteurs & 876 & 8 \\
Auteurs du multimédia interactif*** & 270 & 2 \\
Auteurs de logiciels & 146 & 1 \\
Auteurs d'œuvres dramatiques & 132 & 1 \\
Chorégraphes & 9 & - \\
Ensemble & $\mathbf{1 1 0 1 8}$ & $\mathbf{1 0 0}$ \\
& & \\
\hline $\begin{array}{l}\text { Champ : artistes auteurs affiliés à l'Agessa en 2008, sur la base des reve- } \\
\text { nus d'auteur perçus en 2007. } \\
* * \text { Nomenclature établie par l'Agessa. } \\
\text { *** Le multimédia interactif est une discipline introduite en 2002. }\end{array}$ \\
\hline
\end{tabular}

Source : Agessa/DEPS, 201

Si la traduction est une discipline aux deux tiers féminine, toutes les autres sont majoritairement masculines, dans des mesures variables : en 2008, les femmes représentent entre $40 \%$ et $50 \%$ des écrivains et des auteurs

* Cette étude a bénéficié du concours actif de Pascal Murgier, Thierry Dumas et Samba Diagne, respectivement chef du département des artistes et des professions à la Direction générale de la création artistique du ministère de la Culture et de la Communication, directeur de l'Agessa et chef de projet informatique à l'Agessa. Qu'ils soient ici vivement remerciés.

** Attachée statisticienne de l'Insee, chargée d'analyse au DEPS.

1. En 2006, la catégorie des auteurs d'œuvres cinématographiques (200 en 2005) a été agrégée à celle des auteurs d'œuvres audiovisuelles. Les auteurs d'œuvres audiovisuelles (y compris cinématographiques) sont des réalisateurs, des scénaristes, des dialoguistes, des sous-titreurs, etc.

2. Selon l'enquête Emploi 2008 de l'Insee.

3. La présente étude porte sur les seuls artistes auteurs affiliés à l'Agessa, avec un bref exposé, en encadré, de la situation des assujettis (précomptés). 


\section{Le régime de sécurité sociale des artistes auteurs affiliés}

Toute diffusion ou exploitation commerciale par un diffuseur ${ }^{1}$ d'une œuvre originale d'un artiste auteur donne lieu au versement d'une rémunération (les droits d'auteur). L'acquisition de l'œuvre (tableau, sculpture, etc.) donne également lieu à rémunération. Lorsque l'artiste auteur exerce son activité de façon indépendante (non salariée) et qu'il réside fiscalement en France, le versement des droits d'auteur par le diffuseur ou la vente d'œuvres s'accompagnent du prélèvement de cotisations et contributions obligatoires, opération appelée précompte. Ces cotisations et contributions sont collectées par deux associations agréées:

- la Maison des artistes pour les œuvres d'arts graphiques et plastiques;

- l'Association pour la gestion de la sécurité sociale des auteurs, Agessa, pour les activités de création littéraire, dramatique, musicale, audiovisuelle et photographique.

Les cotisations aux assurances sociales (vieillesse, maladie, maternité, invalidité, décès), la CSG et la CRDS sont dues par l'artiste auteur ${ }^{2}$ au titre du régime de protection sociale des artistes auteurs (articles L.382-1 à L.382-14 et R.382-1 à R.382-36 du Code de la sécurité sociale); ce régime spécifique est rattaché au régime général des salariés.

Sous conditions de ressources et s'il en fait la demande, l'artiste auteur est affilié à la Maison des artistes ou à l'Agessa ; à ce titre, il bénéficie des prestations équivalentes à celles du régime général (indemnités journalières, congés maternité, etc.). Pour cela, il doit avoir perçu au cours de l'année civile précédente des revenus d'auteur ${ }^{3}$ supérieurs au seuil d'affiliation, soit 900 fois la valeur du smic horaire (7524 euros de revenus sur l'année 2007, 7749 euros de revenus sur l'année 2008). Lorsque le seuil d'affiliation n'est pas atteint, l'affiliation ou son maintien peut être prononcé, à titre dérogatoire, par la commission professionnelle de la Maison des artistes ou de l'Agessa, qui juge de l'engagement professionnel de l'artiste auteur. S'il n'est pas affilié, il est dit assujetti (encadré, p. 6).

1. Par exemple, les galeries d'art, les éditeurs, les producteurs de films. Les sociétés d'auteurs (telles que la Sacem, la Scam, la SACD) reversent à l'Agessa les cotisations afférentes aux droits d'auteur qu'elles répartissent. 2. Le diffuseur quant à lui verse à l'Agessa ou à la Maison des artistes une contribution représentant $1 \%$ de la rémunération versée, $1 \%$ des commissions perçues (sociétés de ventes volontaires) ou $1 \%$ de $30 \%$ du chiffre d'affaires issu de la vente d'œuvres artistiques.

3. On entend par revenus d'auteur les revenus imposables au titre des bénéfices non commerciaux majorés de $15 \%$, ou les droits d'auteur bruts s'ils sont déclarés en traitements et salaires.

d'œuvres audiovisuelles, autour de $35 \%$ des illustrateurs mais moins de $20 \%$ des photographes, seulement $10 \%$ des auteurs de compositions musicales et $4 \%$ des auteurs de logiciels (graphique 2).

Minoritaires, les femmes représentent cependant une part croissante des affiliés (28\% des artistes auteurs en 1994, $33 \%$ quinze ans plus tard). Cette féminisation est sensible surtout parmi les écrivains, les auteurs d'œuvres audiovisuelles et dramatiques et les photographes. Dans les autres disciplines, leur part est relativement stable.

\section{Graphique 1 - Évolution du nombre d'artistes auteurs affiliés à l'Agessa selon la discipline, 1994-2008}

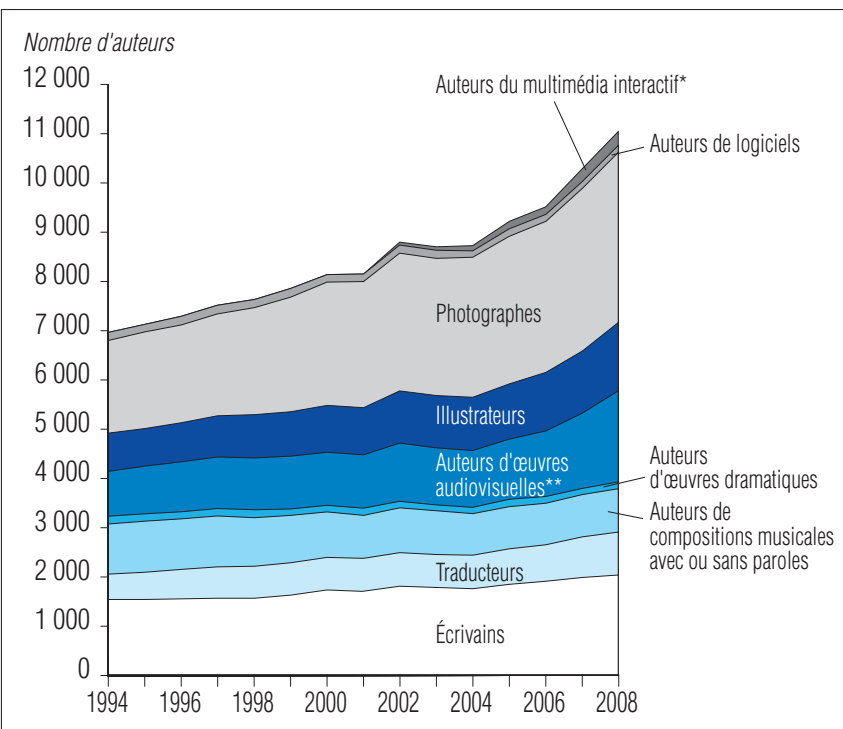

Champ : artistes auteurs affiliés à l'Agessa, de 1994 à 2008. Les effectifs d'une année sont relatifs aux revenus d'auteur perçus l'année précédente.

* Le multimédia interactif est une discipline introduite en 2002.

** $Y$ compris les œuvres cinématographiques.

Note de lecture: en 1994, l'Agessa comptait 1530 écrivains, elle en dénombre 2038 en 2008.

Source : Agessa/DEPS, 2011

\section{Graphique 2 - Les femmes parmi les artistes auteurs affiliés à l'Agessa selon la discipline, 1994, 2001 et 2008}

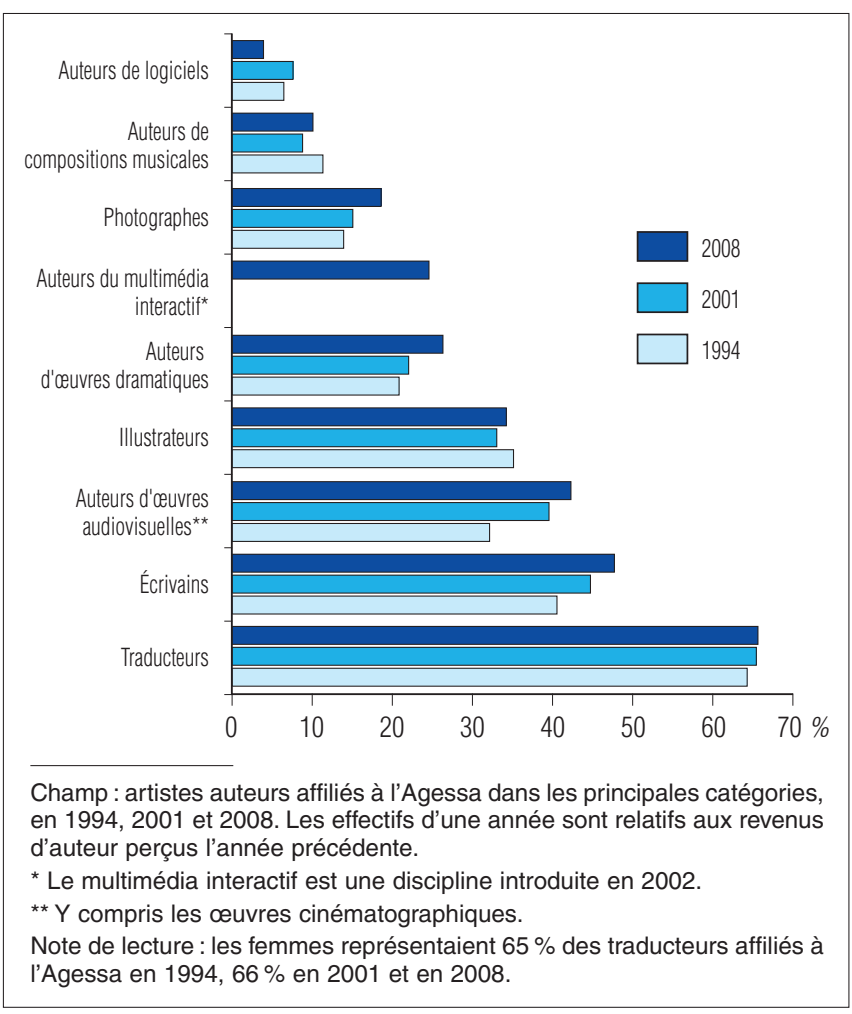

Source : Agessa/DEPS, 2011 


\section{Une population relativement âgée, mais qui rajeunit}

Les artistes auteurs affiliés à l'Agessa sont âgés en moyenne de 49 ans, soit huit ans de plus que l'âge moyen des actifs occupant un emploi en $2008^{4}$. Seulement $23 \%$ ont moins de 40 ans, contre $46 \%$ dans l'ensemble de la population active en emploi.

Les auteurs du multimédia interactif sont, de façon générale, beaucoup plus jeunes que leurs confrères des autres disciplines : ils ont en moyenne 39 ans en $2008^{5}$ (graphique 3). $60 \%$ des auteurs de cette discipline ont moins de 40 ans, et $9 \%$ ont moins de 30 ans.

Les illustrateurs ont en moyenne 45 ans; l'âge moyen des auteurs de logiciels et d'œuvres audiovisuelles ainsi que des photographes et des traducteurs s'établit entre 45 et 50 ans.

Les écrivains et les auteurs de compositions musicales ont en moyenne autour de 52 ans et $20 \%$ d'entre eux ont moins de 40 ans en 2008. Les auteurs d'œuvres dramatiques sont les plus âgés (57 ans en moyenne), avec $41 \%$ d'auteurs de plus de 60 ans et seulement $9 \%$ de moins de 40 ans.

Quinze ans plus tôt, la population des artistes auteurs affiliés à l'Agessa était, globalement et en moyenne, âgée

\section{Graphique 3 - Âge moyen des artistes auteurs affiliés à l'Agessa selon la discipline, 1994, 2001 et 2008}

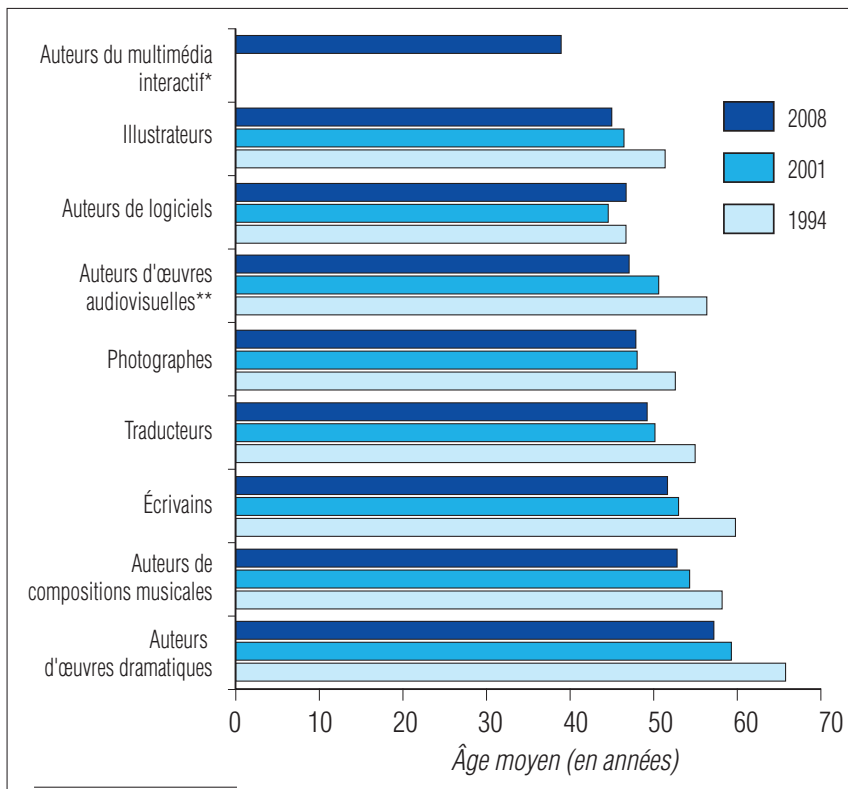

Champ : artistes auteurs affiliés à l'Agessa, en 1994 et 2008. Les effectifs d'une année sont relatifs aux revenus d'auteur perçus l'année précédente.

* Le multimédia interactif est une discipline introduite en 2002.

${ }^{* \star} Y$ compris les œuvres cinématographiques.

Note de lecture: les auteurs d'œuvres dramatiques étaient âgés en moyenne de 66 ans en 1994, 59 ans en 2001 et 57 ans en 2008. de 56 ans. Ce rajeunissement a concerné l'ensemble des disciplines ${ }^{6}$ et particulièrement les auteurs d'œuvres dramatiques et audiovisuelles.

\section{L'Île-de-France moins prépondérante}

En 2008, $38 \%$ des artistes auteurs habitent Paris intramuros et $61 \%$ l'île-de-France ${ }^{7}$.

La surreprésentation des franciliens varie de $40 \%$ à $80 \%$ selon les disciplines : elle s'établit entre $70 \%$ et $80 \%$ parmi les auteurs d'œuvres audiovisuelles, musicales et dramatiques, autour de $60 \%$ pour les photographes, écrivains et traducteurs. La moitié des auteurs de logiciels et du multimédia interactif et $40 \%$ des illustrateurs résident en Île-de-France.

Le poids de l'Île-de-France, en termes d'effectifs, tend toutefois à se réduire puisque $68 \%$ des artistes auteurs y résidaient en 1994 (44\% dans la ville de Paris). Ce mouvement de retrait est particulièrement accentué pour les traducteurs, illustrateurs et auteurs de logiciels et beaucoup plus modéré pour les auteurs d'œuvres audiovisuelles et musicales, qui demeurent massivement franciliens (graphique 4).

\section{Graphique 4 - Répartition géographique des artistes auteurs affiliés à l'Agessa selon la discipline, 1994 et 2008}

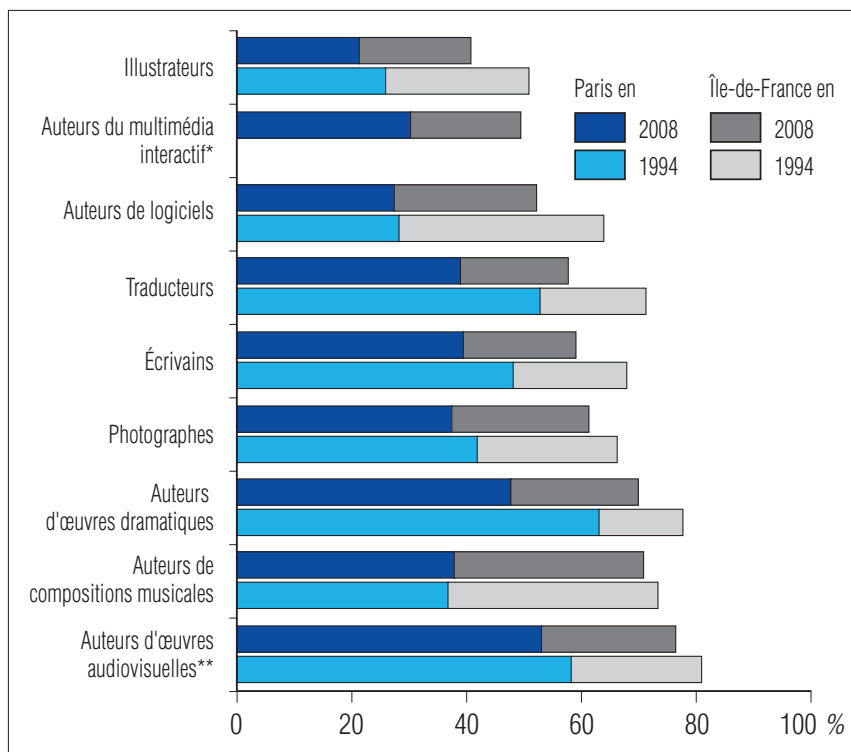

Champ : artistes auteurs affiliés à l'Agessa en 1994 et 2008. Les effectifs d'une année sont relatifs aux revenus d'auteur perçus l'année précédente. * Le multimédia interactif est une discipline introduite en 2002.

${ }^{* *} Y$ compris les œuvres cinématographiques.

Note de lecture : parmi les auteurs d'œuvres audiovisuelles, la proportion de résidents en Île-de-France était de $81 \%$ en 1994 (dont $58 \%$ à Paris intra-muros) et de $76 \%$ en 2008 ( $53 \%$ à Paris).

4. L'âge moyen dans la population française active occupée est de 41 ans (enquête Emploi 2008, Insee). Les graphistes, peintres, sculpteurs et autres artistes affiliés à la Maison des artistes en 2009 sont âgés en moyenne de 44 ans (Maison des artistes/DEPS, 2011).

5. Parmi les artistes auteurs affiliés à la Maison des artistes en 2009, les plus jeunes sont les graphistes, âgés en moyenne de 38 ans.

6. À l'exception du multimédia interactif, qui n'a été introduit qu'en 2002.

7. En 2008, 21 \% des personnes occupant un emploi en France vivent dans la région parisienne (enquête Emploi, Insee). Les peintres, graphistes, sculpteurs et autres artistes auteurs affiliés à la Maison des artistes en 2009 sont $31 \%$ à vivre à Paris intra-muros et $53 \%$ en Île-de-France (Maison des artistes/DEPS, 2011). 


\section{Des revenus d'auteur ${ }^{8}$ très dispersés}

En 2008, près de 5800 artistes auteurs déclarent fiscalement des droits en traitements et salaires et 5850 en bénéfices non commerciaux ${ }^{9}$ (BNC) (tableau 2).

Les auteurs d'œuvres dramatiques, audiovisuelles, musicales ainsi que les écrivains et traducteurs déclarent le plus souvent leurs droits en salaires, tandis que les photographes, les auteurs de logiciels et du multimédia interactif déclarent surtout des bénéfices non commerciaux. Les illustrateurs quant à eux déclarent leurs droits d'auteur dans l'une ou l'autre catégorie de revenus.

Le revenu artistique annuel moyen des catégories d'auteurs déclarant leurs droits en traitements et salaires est sensiblement plus élevé que celui des catégories déclarant en BNC : près de 40000 euros en moyenne pour les premiers en 2008, 29000 euros pour les seconds ${ }^{10}$.

Ces moyennes recouvrent de très fortes disparités d'une discipline à l'autre : les auteurs de compositions musicales déclarent en traitements et salaires des montants près de deux fois plus élevés que les écrivains, et près de trois fois plus importants que les traducteurs. Parmi les disciplines

\section{Tableau 2 - Déclaration en salaires et en BNC des revenus d'auteur perçus selon la discipline en 2007}

\begin{tabular}{|c|c|c|c|}
\hline & \multirow[b]{2}{*}{$\%$} & \multicolumn{2}{|c|}{ Revenus } \\
\hline & & $\begin{array}{c}\text { moyens } \\
(e n €)\end{array}$ & $\begin{array}{c}\text { médians } \\
(e n \in)\end{array}$ \\
\hline \multicolumn{4}{|l|}{ Déclaration en salaires } \\
\hline Auteurs d'œuvres dramatiques & 92 & 56209 & 18125 \\
\hline Écrivains & 86 & 33810 & 16333 \\
\hline Traducteurs & 86 & 24099 & 17554 \\
\hline \multicolumn{3}{|l|}{ Auteurs de compositions musicales } & 24059 \\
\hline \multicolumn{4}{|l|}{ Auteurs d'œuvres } \\
\hline audiovisuelles & 81 & 52931 & 27675 \\
\hline Illustrateurs & 37 & 25333 & 13563 \\
\hline Ensemble & 52 & 39754 & 17883 \\
\hline \multicolumn{4}{|l|}{ Déclaration en BNC } \\
\hline Photographes & 99 & 31459 & 14545 \\
\hline Auteurs du multimédia interactif* & 81 & 19098 & 12786 \\
\hline Auteurs de logiciels & 81 & 67934 & 36667 \\
\hline Illustrateurs & 65 & 26343 & 12171 \\
\hline Ensemble & 53 & 29164 & 12730 \\
\hline \multicolumn{4}{|c|}{$\begin{array}{l}\text { Champ: artistes auteurs affiliés à l'Agessa en } 2008 \text { (revenus d'auteur de } \\
\text { 2007). } \\
\text { * Le multimédia interactif est une discipline introduite en } 2002 \text {. } \\
\text { Note de lecture : en } 2008,86 \% \text { des écrivains déclarent leurs droits d'au- } \\
\text { teur perçus l'année précédente en traitements et salaires. Le revenu moyen } \\
\text { associé est de } 33810 \text { euros ( } 35945 \text { euros, en euros constants, en 2005) } \\
\text { et la moitié des écrivains déclarent moins de } 16333 \text { euros. }\end{array}$} \\
\hline
\end{tabular}

déclarant majoritairement les droits d'auteur en BNC, les auteurs de logiciels déclarent, en moyenne, des bénéfices nettement plus élevés que les autres : près de quatre fois plus que les auteurs du multimédia interactif en 2008.

Toutefois, du fait de la grande hétérogénéité des revenus et des écarts très importants d'un auteur à l'autre au sein d'une même discipline artistique, l'étude du seul revenu moyen ne suffit pas pour appréhender la distribution des droits d'auteur, comme en témoigne la comparaison avec le revenu médian, de $40 \%$ à trois fois moindre que le revenu moyen, selon les disciplines.

Le revenu médian perçu en 2007 s'établit globalement à 17900 euros de traitements et salaires et à 12700 euros de bénéfices non commerciaux : qu'il s'agisse de salaires ou de BNC, le revenu médian des auteurs affiliés à l'Agessa est inférieur au salaire annuel net médian des salariés travaillant à temps complet dans le privé et le semi-public, qui s'établit à 19150 euros en 2007.

La courbe de concentration des revenus artistiques des auteurs (graphique 5) illustre ce phénomène général : la moitié des auteurs se partagent moins de $10 \%$ des revenus (salaires et BNC), tandis que les $10 \%$ d'auteurs les mieux rémunérés en perçoivent à eux seuls plus de la moitié. Ce phénomène est d'autant plus caractéristique de ces populations qu'il est remarquablement stable au cours des dernières années.

La situation d'auteur recouvre donc des réalités très contrastées dont les deux pôles extrêmes sont, d'une part,

\section{Graphique 5 - Concentration des revenus des artistes auteurs affiliés en 2008}

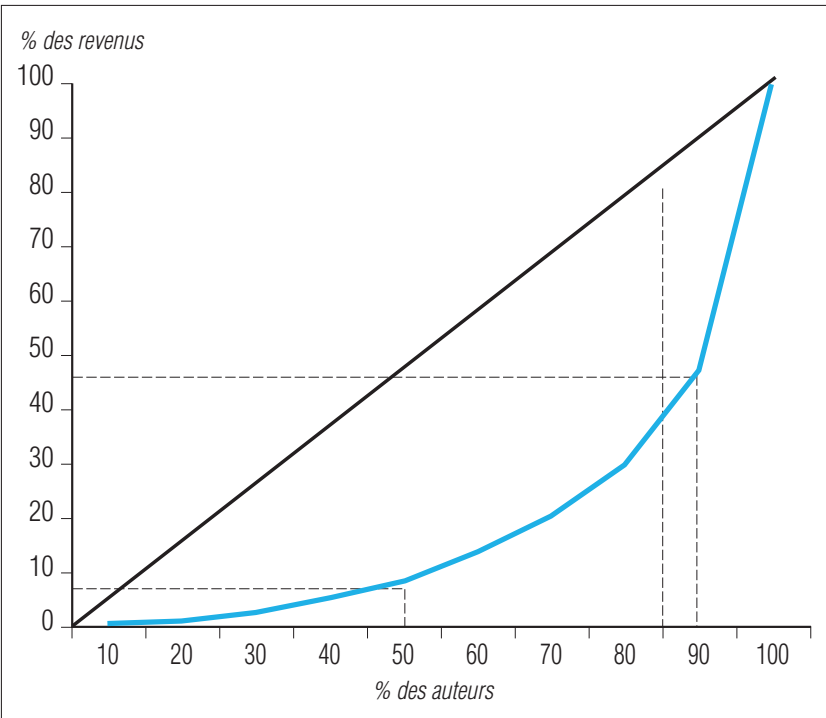

Champ : artistes auteurs affiliés à l'Agessa en 2008, revenus d'auteur de l'année 2007.

Note de lecture : $50 \%$ des artistes auteurs affiliés en 2008 ont déclaré $8 \%$ de l'ensemble des revenus (salaires et BNC).

8. Les sources exploitées couvrent uniquement les revenus issus des activités artistiques et ne tiennent donc pas compte des éventuelles autres sources de revenus des affiliés : elles ne permettent donc pas de mesurer l'ensemble des revenus perçus par les artistes auteurs ni de refléter leur niveau de vie. 9. Plus exactement, près de 5200 déclarent leurs droits d'auteur uniquement en salaires, 5250 uniquement en BNC et 600 auteurs déclarent leurs droits tantôt en salaires, tantôt en BNC.

10. À titre de comparaison, les artistes auteurs affiliés à la Maison des artistes déclarent, en 2009, 24000 euros en moyenne de bénéfices non commerciaux. 


\section{Graphique 6 - Part des revenus d'auteur captés par les $10 \%$ d'auteurs les mieux rémunérés selon la discipline, 2007}

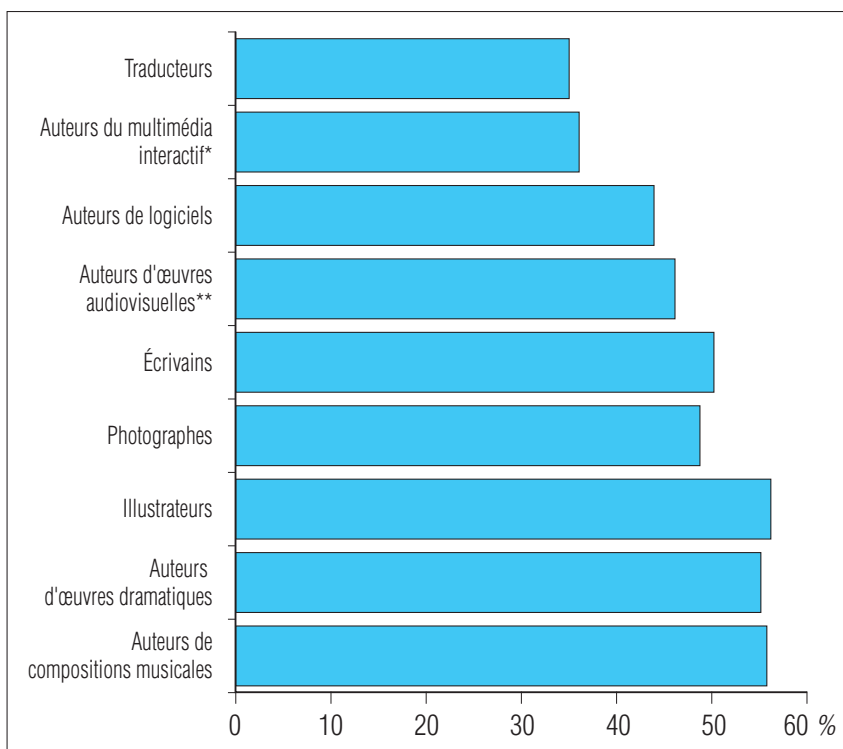

Champ: artistes auteurs affiliés à l'Agessa en 2008, revenus d'auteur de l'année 2007.

* Le multimédia interactif est une discipline introduite en 2002.

${ }^{* *} Y$ compris les œuvres cinématographiques.

Note de lecture: parmi les auteurs de compositions musicales, les $10 \%$ d'auteurs percevant les revenus d'auteur les plus élevés se partagent $56 \%$ des revenus perçus dans la discipline en 2007.

Source : Agessa/DEPS, 2011

un petit nombre d'auteurs parvenant à vivre très largement de leur activité et, de l'autre, une population nombreuse dont les revenus issus de l'activité artistique sont très faibles. Cette caractéristique varie naturellement d'une discipline à l'autre. Elle est particulièrement marquée pour les œuvres dramatiques et musicales où les $10 \%$ d'auteurs les plus favorisés se partagent plus de la moitié des revenus (graphique 6).

Les revenus issus de la traduction et du multimédia interactif sont moins dispersés: les $10 \%$ d'auteurs les mieux rémunérés déclarent $35 \%$ des revenus de ces deux disciplines ${ }^{11}$.

\section{De plus en plus d'artistes auteurs affiliés en deçà du seuil d'affiliation}

En 2008, $29 \%$ des artistes auteurs affiliés à l'Agessa déclarent avoir perçu l'année précédente des revenus inférieurs au seuil d'affiliation ${ }^{12}$. Moins de $25 \%$ des traduc- teurs, des auteurs de logiciels et d'œuvres audiovisuelles sont affiliés en deçà du seuil, tandis que cette proportion s'établit entre $30 \%$ et $35 \%$ pour les auteurs d'œuvres dramatiques, de compositions musicales et du multimédia interactif, des photographes et des illustrateurs.

Cette part a augmenté entre 1994 et 2008 pour la plupart des disciplines et, en particulier, pour les photographes, les illustrateurs et les auteurs d'œuvres dramatiques (graphique 7).

Les auteurs de logiciels, dont les revenus ont sensiblement augmenté au cours de la période, sont les seuls à ne pas s'inscrire dans ce mouvement d'affiliation croissante en deçà du seuil. Le multimédia interactif - très concerné par ce phénomène dans les années qui ont suivi son introduction dans la nomenclature de l'Agessa - s'inscrit désormais en retrait de ce mouvement tout en demeurant à un niveau très élevé d'affiliation en deçà du seuil (un tiers des affiliés sont dans ce cas en 2008).

\section{Graphique 7 - Part des artistes auteurs déclarant des revenus inférieurs au seuil d'affiliation selon la discipline, 1994, 2001, 2005 et 2008}

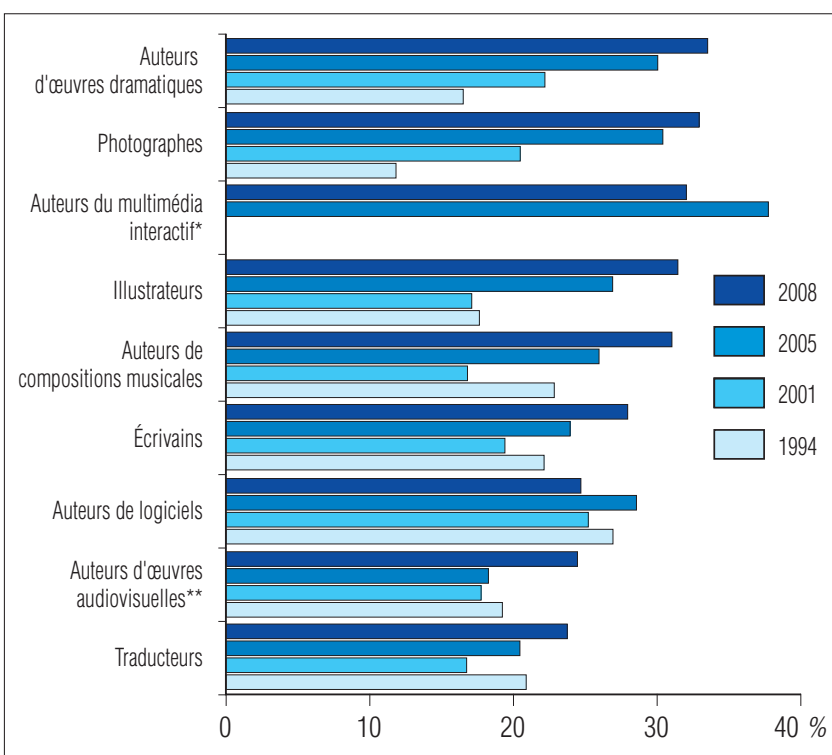

Champ : artistes auteurs affiliés à l'Agessa en 1994, 2001, 2005 et 2008 (sur la base des revenus d'auteur perçus l'année précédente). * Le multimédia interactif est une discipline introduite en 2002.

${ }^{* *} Y$ compris les œuvres cinématographiques.

Note de lecture: les photographes déclarant des revenus d'auteur inférieurs au seuil d'affiliation représentent $12 \%$ des photographes affiliés en 1994 et $33 \%$ en 2008 .

11. Ces distributions sont proches de celles observées deux ans plus tôt, à l'exception de celle relative aux auteurs de logiciels, pour lesquels les revenus se sont globalement élevés et homogénéisés : $10 \%$ des auteurs déclaraient avoir perçu $56 \%$ des revenus en 2005 et $44 \%$ en 2007.

12. Il peut s'agir d'artistes auteurs dont l'affiliation a été prononcée à l'issue d'un examen de leur dossier individuel par la commission professionnelle de l'Agessa ou qui ont été affiliés au-dessus du seuil mais dont les revenus ont baissé depuis l'affiliation. 


\section{Précomptés : les artistes auteurs qui cotisent à l’Agessa sans y être affiliés}

Les cotisations et contributions des artistes auteurs sont prélevées à la source sur leurs droits d'auteur par les diffuseurs ou sociétés d'auteurs ; ces derniers les reversent à l'Agessa : les cotisations et contributions sont ainsi dites précomptées ${ }^{1}$. Concernant les précomptés, c'està-dire les artistes auteurs qui cotisent à l'Agessa sans y être affiliés, la seule information dont l'organisme dispose est contenue dans les précomptes déclarés par les diffuseurs².

De nombreux bordereaux de précompte sont remplis de façon incomplète par les diffuseurs, notamment pour la nature de l'activité des artistes auteurs ; par exemple, plus de 50000 artistes auteurs sont déclarés en tant qu'« auteurs ", 36000 en tant qu' " autres ", sans plus de précision. Ainsi, l'étude des effectifs et des revenus des précomptés à l'Agessa ne peut être détaillée et demeure fragile.

En 2008, l'Agessa dénombre près de 230000 précomptés $^{3}$ (tableau). Lorsque la discipline est identifiée, les auteurs sont le plus souvent déclarés par leurs diffuseurs en tant qu'écrivains (54000), compositeurs (43000) ou photographes (13000). Un peu plus d'un tiers résident en Île-de-France.

En 2008, $12 \%$ des artistes auteurs relevant de l'Agessa ne bénéficiant pas du régime de sécurité sociale des artistes auteurs perçoivent des revenus artistiques supérieurs au seuil d'affiliation (soit plus de 7749 euros); ils sont $9 \%$ parmi les auteurs déclarés comme auteurs compositeurs et $10 \%$ parmi les auteurs déclarés par les diffuseurs en tant qu'écrivains.

Artistes auteurs assujettis à l'Agessa en 2008

\begin{tabular}{|c|c|c|c|c|c|c|c|}
\hline & \multicolumn{3}{|c|}{ Effectifs } & \multicolumn{4}{|c|}{ Revenus d'auteurs (en $€$ ) } \\
\hline & Effectifs & $\%$ & $\begin{array}{c}\% \\
\text { Île-de-France }\end{array}$ & $\begin{array}{l}\text { Moins de } \\
4000\end{array}$ & $\begin{array}{l}\text { De } 4000 \\
\text { à } 7749^{*}\end{array}$ & $\begin{array}{l}\text { De } 7749^{*} \\
\text { à } 33276^{* *}\end{array}$ & $\begin{array}{l}\text { Plus de } \\
33276^{\star \star}\end{array}$ \\
\hline Auteurs-compositeurs & 43331 & 19 & 23 & 86 & 5 & 6 & 3 \\
\hline Écrivains & 53605 & 24 & 39 & 83 & 7 & 8 & 2 \\
\hline Photographes & 12910 & 6 & 40 & & & & \\
\hline Autres disciplines & 29259 & 13 & 46 & & & & \\
\hline «Auteurs » & 52872 & 23 & 41 & & & & \\
\hline «Autres» & 35961 & 16 & 35 & & & & \\
\hline Ensemble & 227938 & 100 & 37 & 81 & 7 & 9 & 3 \\
\hline \multicolumn{8}{|c|}{$\begin{array}{l}\text { Champ : artistes auteurs précomptés à l'Agessa au cours de l'année } 2008 \text { (revenus d'auteur perçus en 2008). } \\
\text { * } 7749 \text { euros correspond au seuil pour une affiliation au régime de sécurité sociale des artistes auteurs en } 2008 . \\
\text { * } 33276 \text { euros correspond au plafond de ressources de la sécurité sociale pour le calcul des cotisations d'assurance vieillesse en } 2008 . \\
\text { Note de lecture : en } 2008 \text {, l'Agessa dénombre } 227938 \text { précomptés; parmi eux, } 37 \% \text { sont franciliens. Les écrivains représentent } 24 \% \text { des précomptés en } \\
2008 \text { et, parmi eux, } 39 \% \text { résident en île-de-France. Cette année-là, } 12 \%(9 \%+3 \% \text { ) des précomptés perçoivent des revenus d'auteur supérieurs au seuil } \\
\text { d'affiliation ( } 7749 \text { euros ou plus) ; ils sont } 10 \% \text { parmi les auteurs déclarés comme écrivains, et } 9 \% \text { parmi les auteurs-compositeurs. }\end{array}$} \\
\hline
\end{tabular}

Source : Agessa/DEPS, 2011

1. Il s'agit des cotisations aux assurances sociales (hormis l'assurance vieillesse), de la csG et de la cRDs. Les cotisations d'assurance vieillesse ne peuvent faire l'objet d'un précompte. En effet, seule la connaissance de l'ensemble des revenus soumis à cotisation vieillesse permettrait d'appeler ces cotisations dans la limite du plafond de la sécurité sociale.

2. Pour les affiliés, l'Agessa dispose de davantage d'informations, ceux-là devant s'identifier auprès de l'organisme lorsqu'ils demandent leur affiliation.

À la Maison des artistes, tous les artistes auteurs, qu'ils soient affiliés ou assujettis, s'identifient auprès de l'organisme lorsqu'ils débutent leur activité. Un an après avoir débuté leur activité, ils peuvent opter pour la dispense de précompte. De plus, les ventes d'œuvres à des particuliers ou par l'intermédiaire des galeries ne font pas l'objet de précompte, l'artiste se chargeant de les déclarer à la Maison des artistes l'année qui suit la perception des revenus. En définitive, le mécanisme du précompte concerne principalement les dessinateurs textile et les graphistes, et pour leur première année d'activité. À l'inverse, sauf à demander leur affiliation, les précomptés relevant de l'Agessa continuent de voir leurs cotisations et contributions sociales prélevées à la source.

3. La Maison des artistes compte un peu plus de 20000 artistes auteurs assujettis en 2009. 


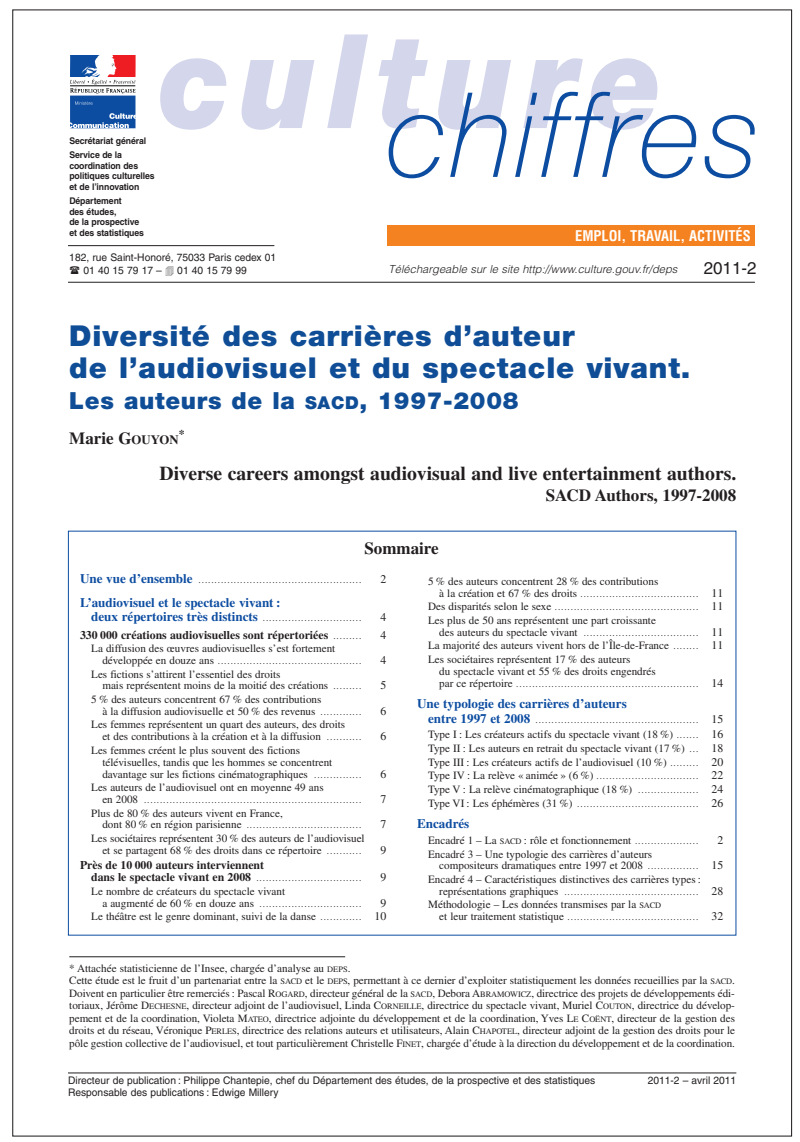

\section{Marie GouYoN}

\section{Diversité des carrières d'auteur de l'audiovisuel et du spectacle vivant. Les auteurs de la SACD, 1997-2008}

Paris, DEPS, Ministère de la Culture et de la Communication, coll. "Culture chiffres », 2011-2, avril 2011

En 2008, la Société des auteurs et compositeurs dramatiques (SACD) a versé 136 millions d'euros de droits à 17700 auteurs et compositeurs dramatiques. Entre 1997 et 2008, les répertoires gérés par la SACD ont beaucoup évolué, en particulier celui de l'audiovisuel, du fait des mutations technologiques intervenues depuis la fin des années 1990 dans ce secteur : apparition puis généralisation de la télévision numérique terrestre (TNT), développement des chaînes thématiques, etc.

Les auteurs se distinguent nettement les uns des autres selon le répertoire (audiovisuel ou spectacle vivant) auquel ils participent, leur durée et leur volume de production, leur niveau de contributions aux diffusions et aux représentations, ainsi par les montants de droits perçus. La population des auteurs et compositeurs dramatiques reste âgée et très masculine, bien que les femmes y soient de plus en plus nombreuses.

\section{Marie GouYon \\ Peintres, graphistes, sculpteurs... Les artistes auteurs affiliés
à la Maison des artistes
en 2009}

Peintres, graphistes, sculpteurs... Les artistes auteurs affiliés à la Maison des artistes en 2009 Marie Gouyon*

Painters, graphic designers, sculptors, etc. Artist-author members of the Maison des Artistes in 2009

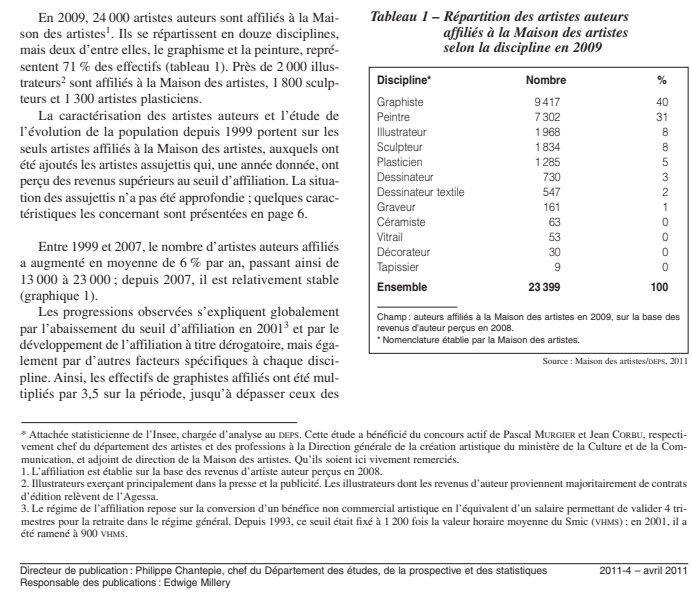

Paris, DEPS, Ministère de la Culture et de la Communication, coll. "Culture chiffres », 2011-4, avril 2011

En 2009, la Maison des artistes affilie 24000 artistes auteurs, principalement graphistes et peintres. Leur nombre s'est élevé en moyenne de 6\% par an entre 1999 et 2006 et est relativement stable depuis 2007.

Près de $60 \%$ des artistes auteurs affiliés sont des hommes. Toutefois, les femmes sont de plus en plus nombreuses, en particulier parmi les plasticiens, les peintres et les graveurs. Les artistes auteurs sont âgés en moyenne de 44 ans et vivent pour plus de la moitié en Île-de-France.

La moitié se partage $15 \%$ de l'ensemble des revenus d'artistes auteurs affiliés à la Maison des artistes, tandis que les $10 \%$ d'artistes auteurs les mieux rémunérés concentrent à eux seuls $43 \%$ de ces revenus. 


\section{RÉSUMÉ}

En 2008, 1'Association pour la gestion de la sécurité sociale des auteurs (Agessa) affilie 11000 artistes auteurs, principalement du livre, de la photographie et de l'audiovisuel. Leur nombre s'est élevé de $58 \%$ au cours des quinze dernières années.

Les deux tiers des artistes auteurs affiliés sont des hommes. Toutefois, les femmes sont de plus en plus nombreuses, en particulier parmi les écrivains, les photographes et les auteurs d'œuvres audiovisuelles et dramatiques. La population des artistes auteurs est assez âgée (49 ans en moyenne) et fortement implantée en Île-de-France.

La moitié se partage moins de $10 \%$ de l'ensemble des revenus d'auteurs affiliés à l'Agessa, tandis que les $10 \%$ d'artistes auteurs les mieux rémunérés concentrent à eux seuls plus de la moitié de ces revenus.

\section{ABSTRACT}

In 2008, France's association for managing artist-authors' social security, AGESSA (Association pour la gestion de la sécurité sociale des auteurs) had 11,000 artist-author members, mainly in the books, photography and audiovisual fields. Member numbers have risen $58 \%$ over the last fifteen years.

Two-thirds of artist-author members are men. However, the number of women is increasing, particularly writers, photographers and authors of audiovisual and dramatic works. The artist-author population is relatively old (average age 49), and with strong representation in the Ille de France.

About $50 \%$ of its members share in less than $10 \%$ of AGESSA members' total artist-author revenue, whilst some $10 \%$ of the wealthier artist-authors are responsible for half of this income.

Tous les documents publiés par le DEPS sont téléchargeables sur http://www.culture.gouv.fr/deps Le DEPS n'assurant pas de diffusion physique de ces documents, nous vous proposons de vous informer régulièrement des parutions par message électronique. Pour ce faire, merci de bien vouloir nous communiquer votre courriel à l'adresse contact.deps@culture.gouv.fr 